\title{
Evaluation of Object Tracking System using Open-CV In Python
}

\author{
*Hemalatha Vadlamudi \\ Assistant Professor, \\ Krishna Chaithanya Institute of Science and Technology, \\ Nellore, AP,
}

\begin{abstract}
Object Tracking System used to track the motion trajectory of an object in a video. First, I use the OpenCV's function, select ROI, to select an object on a frame and track its motion using built-in-tracker. Next, Instead of using selectROI, I use YOLO to detect an object in each frame and track them by object centroid and size comparison. Then I combine YOLO detection with the OpenCV's built in tracker by detecting the object in the first frame using YOLO and tracking them using selectROI. The video tracking is widely used in multiple purpose such as: human -computer interaction, security and surveivallence, traffic control, medical imaging, and so on.
\end{abstract}

Keywords:- OpenCV, YOLO, object tracking, centroid tracking, Frame Differencing, Single shot detector, Background subtraction.

\section{INTRODUCTION}

Object tracking is a very challenging task in the presence of variability Illumination condition, background motion, complex object shape partial and full object occlusions. Object detection and location in digital images has become one of the most important applications for industries to ease user, save time and to achieve parallelism. This is not a new technique but improvement in object detection is still required in order to achieve the targeted objective more efficiently and accurately. The main aim of studying and researching computer vision is to simulate the behavior and manner of human eyes directly by using a computer and later on develop a system that reduces human efforts shows the basic block diagram of detection and tracking. In this paper, an SSD and Mobile Nets based algorithms are implemented for detection and tracking in python environment. Object detection involves detecting region of interest of object from given class of image. Different methods are -Frame differencing, Optical flow, Background subtraction. This is a method of detecting and locating an object which is in motion with the help of a camera. Detection and tracking algorithms are described by extracting the features of image and video for security applications. Features are extracted using CNN and deep learning. Classifiers are used for image classification and counting. YOLO based algorithm with GMM model by using the concepts of deep learning will give good accuracy for feature extraction and classification.

\section{OBJECTIVES:}

Object tracking system aims to improve performance of object detection and tracking by contributing originally to two components 1) motion segmentation 2) object tracking

Therefore the main objectives are:

- To identify the targeted object in moving sequence

- To analyze YOLO based algorithm with GMM model to get good accuracy for feature extraction and classification

- To analyze the motion of the object in a video using OpenCV

- To analyze SSD and Mobile Nets algorithm for tracking the objects

\section{REVIEW OF LITERATURE}

In the previous study most of them have concentrated towards object detection (Chandan G, Ayush Jain, Harsh Jain, Mohana, BenAyed etal., 2015; Najva and Bijoy, 2016; Ramya and Rajeswari, 2016; Risha andKumar, 2016; Shen et al., 2013; Soundrapandiyan and Mouli, 2015; Viswanath et al.,2015) ,Object tracking (Bagherpour et al., 2012; Coşkun and Ünal, 2016; Foytik et al.,2011; Lee et al., 2012; Poschmann et al.,2014; Weng et al., 2013; Yilmaz et al., 2006;Zhang et al., 2016) and Object recognition (Chakravarthy et al., 2015; Elhariri et al.,2015; Gang et al., 2010; Ha and Ko, 2015; Nair et al., 2011) for tracking the object using vedio sequence . There is no object detection in existing system by using Opencv. It is difficult to classify the moving object tracking. The accuracy of object detection is very low.But in my Object tracking system I am using YOLO based GMM model and OpenCV to improve the accuracy of the Object in vedio and also It can easily track the moving object by using point tracking and kernel tracking methods.It not only detect the object but also track the object in the videos.

\section{DESIGN AND DEVELOPMENT}

The most creative and challenging phase of the life cycle is system and design. The term design describes a final system and the process by which it is developed. It refer to the technical specifications that will be applied in implementation the candidate system. The design may be defined as "the process of applying various techniques and principles for the purpose of defining a device, a process or a system in sufficient details to permit its physical realization". 


\section{V- MODEL DESIGN}

Under V-Model, the corresponding testing phase of the development phase is planned in parallel.

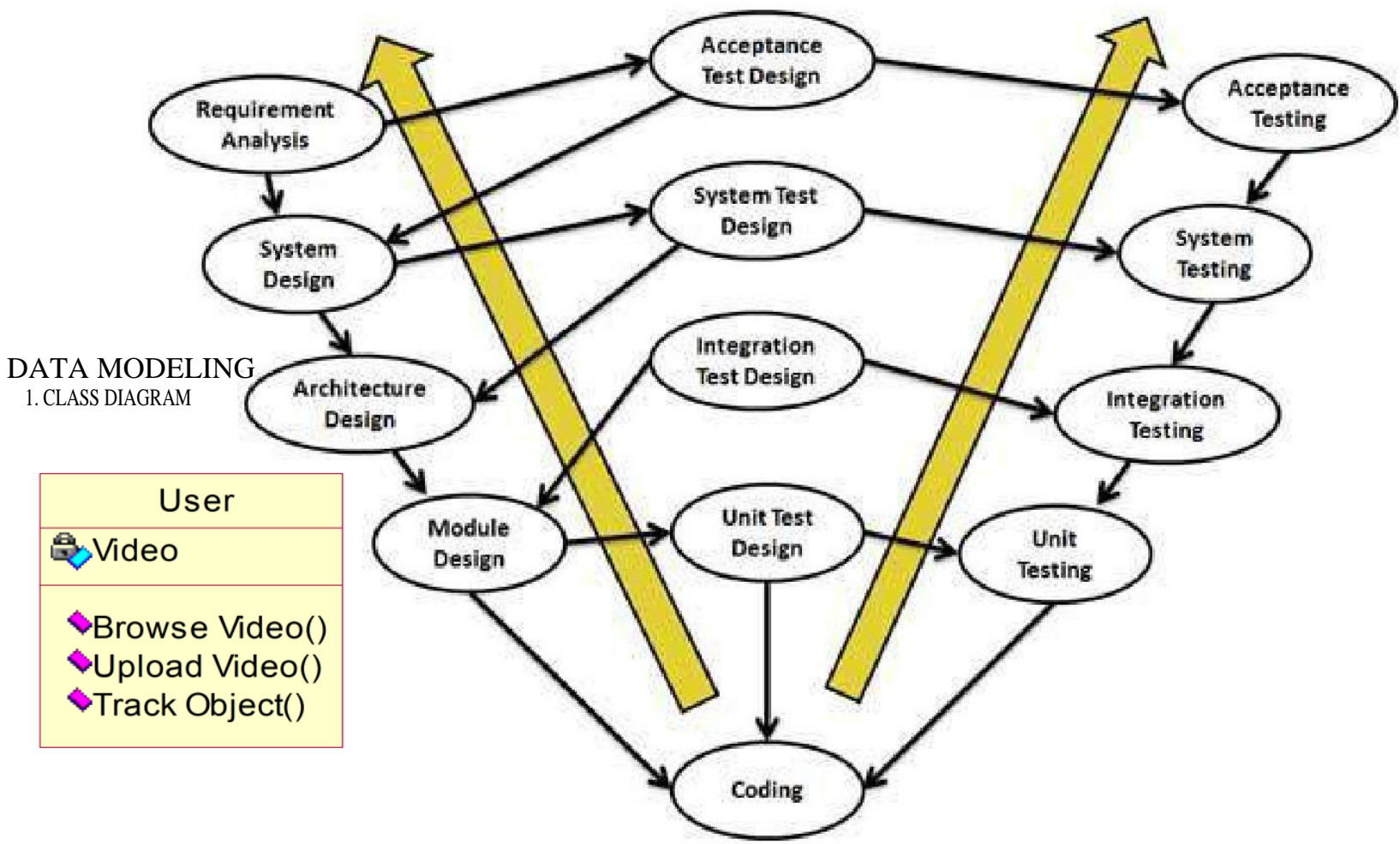

USE CASE DIAGRAM:

A use case diagram in the Unified Modeling Language (UML is a kind of behavioral outline positive by and produced using a Use-contextual investigation. Its determination is to surviving a graphical sign of the usefulness giving by a framework regarding performing artists and their points (spoke to as use cases), and any conditions between those utilization cases. The key reason for an utilization case outline is to show what framework capacities are performed for which on-screen character. Parts of the on-screen characters in the framework can be delineated.

\section{SEQUENCE DIAGRAM:}

\section{Use case}

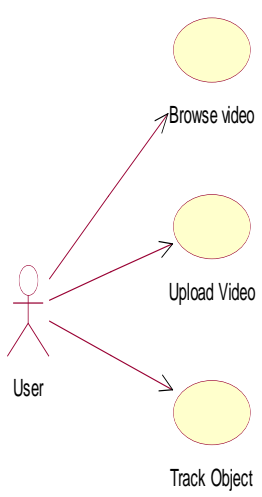

1. SEQUENCE DIAGRAM

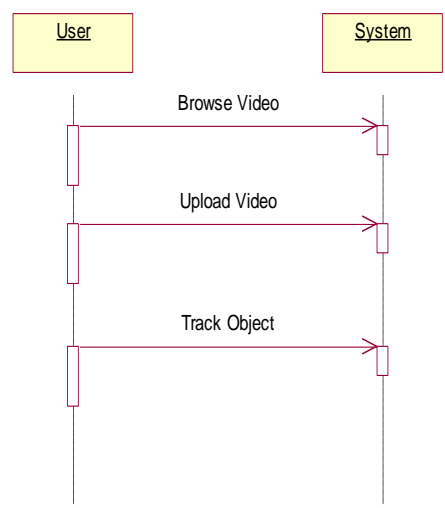

A sequence diagram in Unified Modeling Language (UML) is a kind of interaction diagram that shows how processes operate with one another and in what order. It is a construct of a Message Sequence Chart. Sequence diagrams are sometimes called event diagrams, event scenarios, and timing diagrams. 


\section{MODULES}

Implementation is the stage of the project when the theoretical design is turned out into a working system. Thus it can be considered to be the most critical stage in achieving a successful new system and in giving the user, confidence that the new system will work and be effective.

The implementation stage involves careful planning, investigation of the existing system and it's constraints on implementation, designing of methods to achieve changeover and evaluation of changeover methods.

- Browse System Videos:

- Start Webcam Video Tracking:

Browse System Videos:

\section{MODULE DESCRIPTION:}

Using this module application allow user to upload any video from his system and application will connect to that video and start playing it, while playing if application detect any object then it will mark that object with bounding boxes, while playing video if user wants to stop tracking then he need to press ' $\mathrm{q}$ ' key from keyboard to stop video playing.

Start Webcam Video Tracking:

Using this module application connect itself with inbuilt system webcam and start video streaming, while streaming if application detect any object then it will surround that object with bounding boxes, while playing press ' $q$ ' to stop web cam streaming.

\section{TESTING AND VALIDATION}

The goal of testing is to acquire errors. Testing is that the technique of trying to get each possible error or weakness in an extremely work product. It provides the way to observe the practicality of parts, sub-assemblies, assemblies and or a finished product it is the technique of effort code with the concentrating of guaranteeing that the software meets its requirements and user hopes and does not fail in an undesirable manner. There are numerous sorts of check. Every check sort reports a designated testing demand.

Testing objectives:

The key objective of testing is to determine a mass of errors, systematically and with minimum effort and time. Stating formally, we can say, testing is a process of executing a program with resolved of discover an error.

- A successful test is one that determines an as however undiscovered error.

- A good test case is one that has possibility of discover an error, if it exists.

- The test is insufficient to detect possibly present errors.

- The software more or less approves to the quality and unswerving standards.

Acceptance Testing

User Acceptance Testing is a serious phase of any project and needs important contribution by the end user. It also guarantees that the system encounters the functional requirements.

\begin{tabular}{|c|c|c|c|c|}
\hline Test case id & Test case description & Actual value & Entered value & Status \\
\hline 1 & $\begin{array}{c}\text { Register user details in registration } \\
\text { page }\end{array}$ & $\begin{array}{l}\text { Fill all the fields while } \\
\text { registering user }\end{array}$ & All the fields are filled & Pass \\
\hline 2 & Give user name in text box & $\begin{array}{l}\text { User name must be given in } \\
\text { alphabets }\end{array}$ & $\begin{array}{c}\text { User name given in alphabets } \\
\text { and numeric values }\end{array}$ & Fail \\
\hline 3 & $\begin{array}{l}\text { Password to be entered in password } \\
\text { box }\end{array}$ & $\begin{array}{c}\text { Password must be given } \\
\text { correctly }\end{array}$ & Password is entered wrongly & Fail \\
\hline 4 & $\begin{array}{c}\text { Phone number must be entered in } \\
\text { phone number box during } \\
\text { registration }\end{array}$ & $\begin{array}{c}\text { Phone number must be given } \\
\text { in } 10 \text { digits }\end{array}$ & $\begin{array}{c}\text { Phone number given in } 10 \\
\text { digits }\end{array}$ & Pass \\
\hline 5 & $\begin{array}{l}\text { Validating the functionality of } \\
\text { Browse button }\end{array}$ & $\begin{array}{l}\text { System should select the } \\
\text { corresponding file }\end{array}$ & $\begin{array}{l}\text { selected the file what we } \\
\text { expected }\end{array}$ & Pass \\
\hline
\end{tabular}

Testcase Template

Test Results: All the test cases stated above passed effectively. No defects met. 


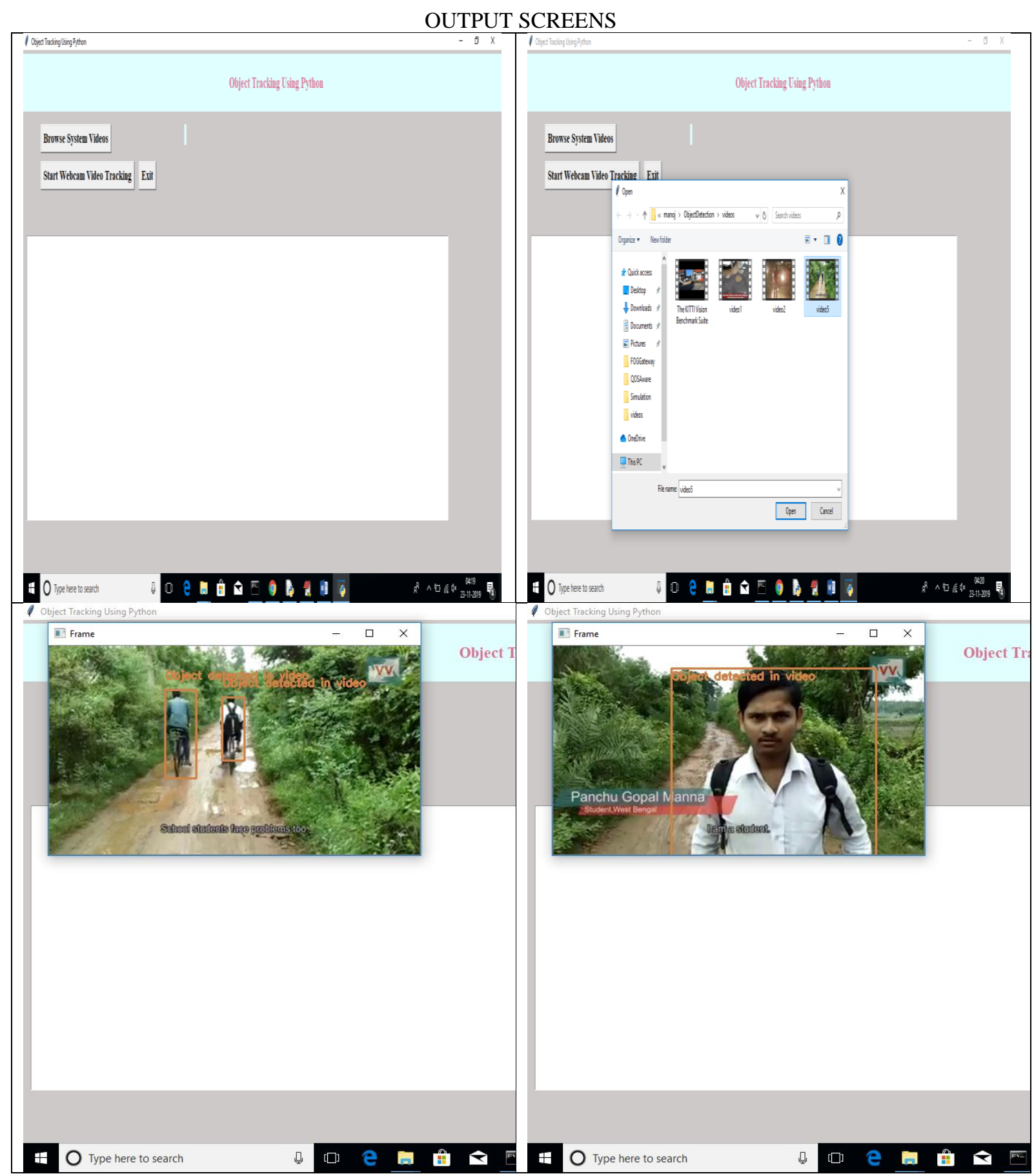




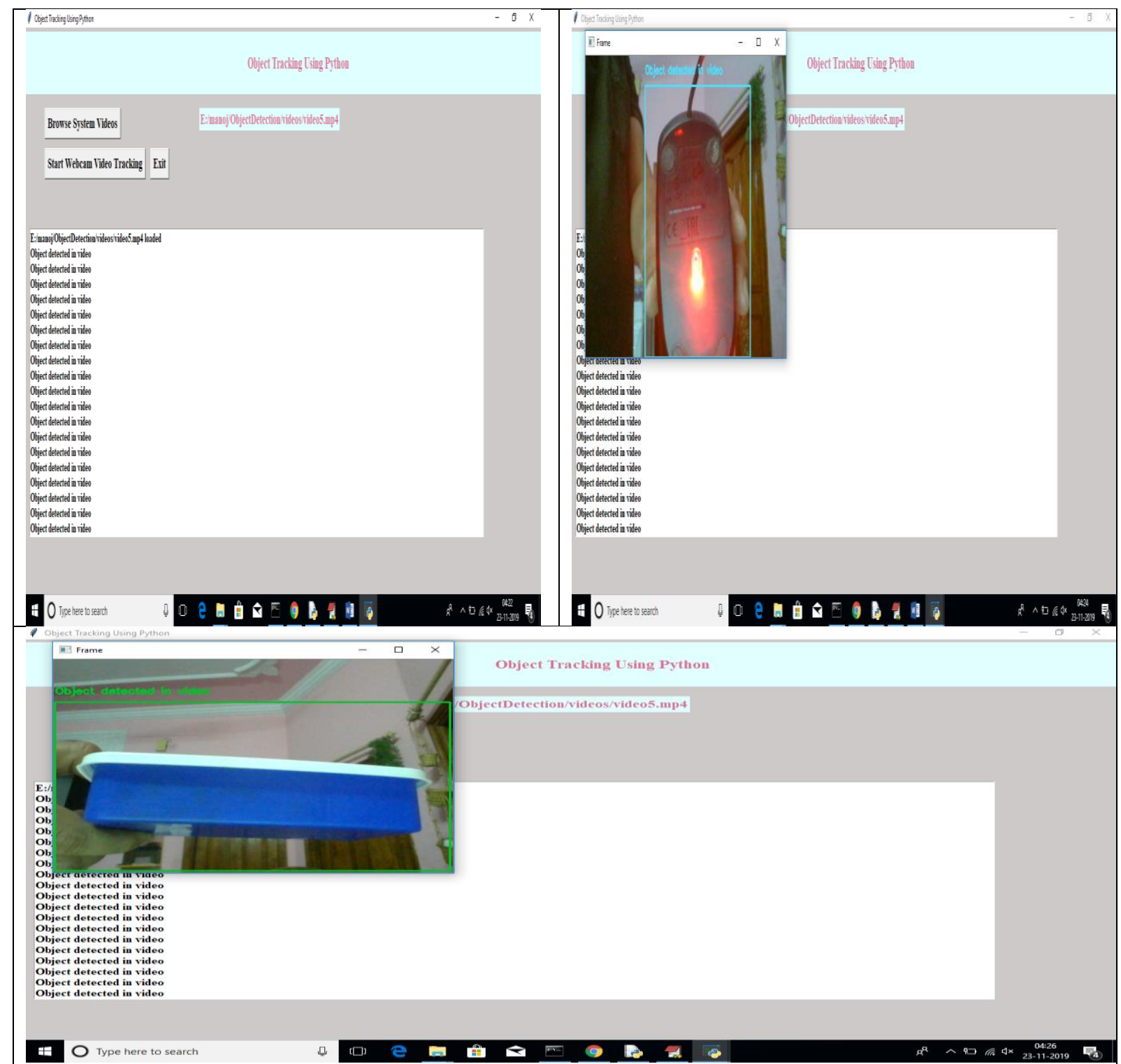

CONCLUSION

An accurate and efficient object detection system has been developed which achieves comparable metrics with the existing state-of-the-art system. This project uses recent techniques in the field of computer vision and deep learning.

\section{SCOPE FOR FUTURE ENHANCEMET}

Although the visual tracking algorithm proposed here is robust in many of the conditions, it can be made more robust by eliminating some of the limitations as listed below:

In the Single Visual tracking, the size of the template remains fixed for tracking. If the size of the object reduces with the time, the background becomes more dominant than the object being tracked. In this case the object may not be tracked.

Fully occluded object cannot be tracked and considered as a new object in the next frame.

To make the system fully automatic and also to overcome the above limitations, in future, multi- view tracking can be implemented using multiple cameras. Multi view tracking has the obvious advantage over single view tracking because of wide coverage range with different viewing angles for the objects to be tracked.

\section{BIBLIOGRAPHY \& REFERENCES REFERENCES}

[1] Wei Liu and Alexander C. Berg, "SSD: Single Shot MultiBox Detector", Google Inc., Dec 2016.

[2] Andrew G. Howard, and Hartwig Adam, "MobileNets: Efficient Convolutional Neural Networks for Mobile Vision Applications", Google Inc., 17 Apr 2017.

[3] Justin Lai, Sydney Maples, “Ammunition Detection: Developing a Real-Time Gun Detection Classifier", Stanford University, Feb 2017

[4] ShreyamshKamate, "UAV: Application of Object Detection and Tracking Techniques for Unmanned Aerial Vehicles", Texas A\&M University, 2015.

[5] Adrian Rosebrock, "Object detection with deep learning and OpenCV", pyimagesearch. 
[6] Mohana and H. V. R. Aradhya, "Elegant and efficient algorithms for real time object detection, counting and classification for video surveillance applications from single fixed camera," 2016 International Conference on Circuits, Controls, Communications and Computing (I4C), Bangalore, 2016, pp. 1-7.

[7] AkshayMangawati, Mohana, Mohammed Leesan, H. V. Ravish Aradhya, "Object Tracking Algorithms for video surveillance applications" International conference on communication and signal processing (ICCSP), India, 2018, pp. 0676-0680.

[8] ApoorvaRaghunandan, Mohana, PakalaRaghav and H. V. Ravish Aradhya, "Object Detection Algorithms for video surveillance applications" International conference on communication and signal processing (ICCSP), India, 2018, pp. 0570-0575.

[9] ManjunathJogin, Mohana, "Feature extraction using Convolution Neural Networks (CNN) and Deep Learning" 2018 IEEE International Conference On Recent Trends In Electronics Information Communication Technology,(RTEICT) 2018, India.

[10] ArkaPrava Jana, AbhirajBiswas, Mohana, "YOLO based Detection and Classification of Objects in video records" 2018 IEEE International Conference On Recent Trends In Electronics Information Communication Technology, 